\title{
Dynamic Manipulation of Cell Membrane Curvature by Light- Driven Reshaping of Azopolymer
}

\author{
Selene De Martino ${ }^{\dagger} \neq, \S$, Wei Zhang ${ }^{\S}$, Lasse Klausen§, Hsin-Ya Lou ${ }^{\S}$, Xiao Li§, Felix S. \\ Alfonso ${ }^{\S}$, Silvia Cavalli ${ }^{\dagger}$, Paolo A. Netti ${ }^{\dagger}, \neq$, Francesca Santoro ${ }^{*}, \dagger$, Bianxiao Cui ${ }^{\star}, \S$ \\ †Center for Advanced Biomaterials for Healthcare, Istituto Italiano di Tecnologia, Largo Barsanti e \\ Matteucci, 53, 80125 Napoli, Italy \\ FDipartimento di Ingegneria Chimica dei Materiali e della Produzione Industriale, DICMAPI, \\ Università degli Studi di Napoli Federico II, Piazzale Tecchio, 80, 80125 Napoli, Italy \\ $\S$ Department of Chemistry, Stanford University, 333 Campus Drive, Stanford, California 94305, \\ United States
}

\begin{abstract}
Local curvatures on the cell membrane serve as signaling hubs that promote curvature-dependent protein interactions and modulate a variety of cellular processes including endocytosis, exocytosis, and the actin cytoskeleton. However, precisely controlling the location and the degree of membrane curvature in live cells has not been possible until recently, where studies show that nanofabricated vertical structures on a substrate can imprint their shapes on the cell membrane to induce well-defined curvatures in adherent cells. Nevertheless, the intrinsic static nature of these engineered nanostructures prevents dynamic modulation of membrane curvatures. In this work, we engineer light-responsive polymer structures whose shape can be dynamically modulated by light and thus change the induced-membrane curvatures on-demand. Specifically, we fabricate threedimensional azobenzene-based polymer structures that change from a vertical pillar to an elongated vertical bar shape upon green light illumination. We observe that U2OS cells cultured on azopolymer nanostructures rapidly respond to the topographical change of the substrate underneath. The dynamically induced high membrane curvatures at bar ends promote local accumulation of actin fibers and actin nucleator Arp $2 / 3$ complex. The ability to dynamically manipulate the membrane curvature and analyze protein response in real-time provides a new way to study curvature-dependent processes in live cells.
\end{abstract}

\section{Graphical Abstract}

\footnotetext{
*Corresponding Authors: bcui@stanford.edu. francesca.santoro@iit.it.

The authors declare no competing financial interest.

Supporting Information

The Supporting Information is available free of charge at https://pubs.acs.org/doi/10.1021/acs.nanolett.9b04307.

Materials and methods; cell viability on azopillars/azobars; membrane curvature modulation on static and dynamic structures (PDF)
} 


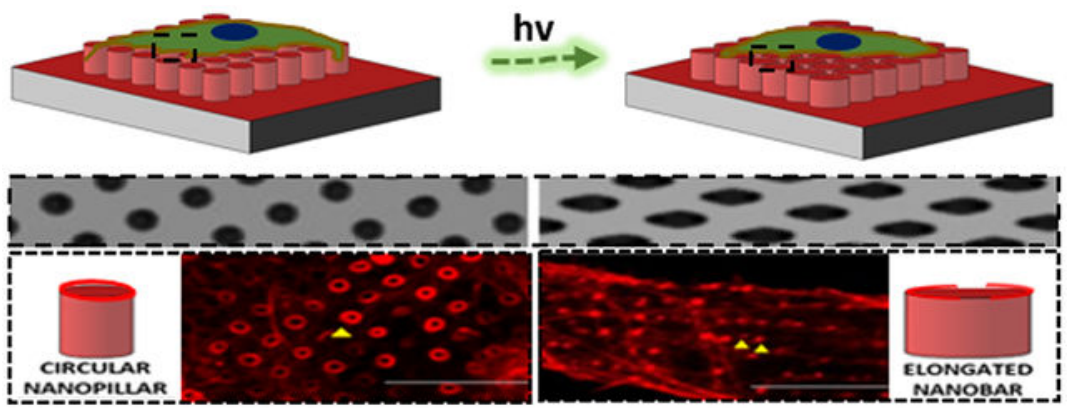

\section{Keywords}

Azopolymer; topography; nanobio interface; light-stimuli materials; F-actin; dynamic biointerface

Many studies show that nanoscale 3D structures significantly affect cell adhesion, migration, proliferation, and stem cell differentiation. ${ }^{1,2} \mathrm{~A}$ few examples include nanopillars enhancing osteogenic differentiation of mesenchymal stem cells (MSCs) ${ }^{3,4}$ and inhibiting cell migration, ${ }^{5,6}$ nanogratings and nanopores inducing cell alignment ${ }^{7-9}$ and differentiation of stem cells, ${ }^{10-13}$ and nanoroughs modulating macrophage activity. ${ }^{14,15}$ At the cellular level, the presence of 3D nanostructures locally changes the curvature of the plasma membrane, which affects curvature-dependent intracellular processes such as endocytosis ${ }^{16,17}$ and actin dynamics. ${ }^{18-22}$ When cells are in contact with 3D structured surfaces, their cell membranes deform to wrap around these structures. The resulting membrane curvatures act as biochemical signals to activate intracellular curvature-dependent proteins and underly $3 \mathrm{D}$ structure-induced regulation and tuning cell behavior. ${ }^{19}$ For example, our recent study shows that 3D nanostructures promote actin polymerization in a curvature-dependent manner and are mediated by a curvature-sensing protein FBP17. ${ }^{23}$

Previous studies of topographic effect on cell behavior use prefabricated and static 3D structures. ${ }^{21,23-28}$ Although these structures were very useful in identifying curvaturedependent protein components and intracellular processes, their static nature means that membrane curvatures induced by these structures are also static. On the other hand, curvatures on the cell membrane appear and disappear dynamically in response to complex intracellular and extracellular cues. Smart materials with the ability to change their 3D shapes upon light stimulation would afford a new approach to manipulate membrane curvatures on-demand. ${ }^{29-32}$

In the last two decades, light-responsive materials such as azobenzene-based polymeric compounds have attracted significant interest as smart materials. The photoisomerization of azobenzene induces conformational changes of the polymer chains that in turn lead to modifications of the material physical and mechanical properties. ${ }^{33}$ For example, a cyclic trans-cis-trans isomerization of azobenzene upon light exposure leads to a mass displacement of azopolymer either at the microscopic or macroscopic level along the polarization direction of the illumination beam. ${ }^{34,35}$ The light-induced deformation of azopolymer has been demonstrated to cause significant shape changes when azobenzenebased polymers are made into 3D structures. ${ }^{29,36,37}$ This process, referred as directional 
photofluidization lithography, has provided advances in the fabrication of complex micro and nano-architectures otherwise not easily fabricated by conventional methods. ${ }^{38}$ Furthermore, light-induced deformation of azopolymer has shown to dynamically change material characteristics such as melting temperature, robustness, and surface wettability. $29,36,37,39-41$

In this work, we utilize light-driven reshaping of azopolymer to dynamically manipulate the geometry of 3D vertical nanostructures. The cell membrane is highly fluid and dynamic so that it easily adapts to mechanical perturbations. Previous work has shown that the cell membrane readily deforms when pushed or pulled by atomic force microscopy or optical tweezers. ${ }^{42,43}$ Therefore, the cell membrane will change shape together with the azopolymer nanostructures upon green light illumination. Here, we show that light-driven reshaping of azopolymer nanopillars to nanobars in the presence of living cells dynamically imparts curvatures at the plasma membrane (PM). The induced membrane curvatures promote the formation of actin fibers at the locations of high curvature and accumulation of the actin nucleator Arp2/3 complex. ${ }^{23,44}$ The ability to manipulate the membrane curvature ondemand and analyze cell response in real-time provides a new way to study how surface topography modulates cell behavior and intracellular processes.

\section{Results.}

\section{Light-Induced Reshaping of Vertical Azopolymer Pillars.}

Our substrates consist of well-ordered arrays of vertical micropillars made of pDR1m azopolymer, fabricated on glass coverslips by soft lithography (through a PDMS mold, which is replicated against a silicon master wafer) as shown in Figure 1a. The fabrication process of the silicon master by e-beam lithography allows for precise control over the pillar dimensions as well as the center-to-center distance (pitch) between pillars. The silicon master was inversely replicated into PDMS molds that were subsequently used as stamps for imprinting the azopolymer pillars. Under polarized light irradiation, the azopolymer of the same polarization absorbs photons, and the trans-cis isomerization induces an athermal transition of the glassy azopolymer into a fluid state. This induces a distinct mass migration along the direction of the light polarization and a shape transition from pillars to ellipsoidal bars. ${ }^{41}$

We first used a $532 \mathrm{~nm} \mathrm{CW}$ linearly polarized solid state laser to illuminate a relatively large area of the substrate. The degree of deformation depends on the irradiation conditions such as wavelength, intensity, and exposure time ${ }^{45}$ Figure $1 \mathrm{~b}$ shows the brightfield images of an azopolymer nanopillar array before and after $5 \mathrm{~s}$ of illumination by the $532 \mathrm{~nm}$ laser at 1.5 $\mathrm{mW}$; the light exposure allows for the transition from circular nanopillars into elongated nanobars of the whole array. The deformation magnitude increases with the exposure time providing a shape progression from pillar to slight elongation, to a bar-like structure in 0,4 , 8 , and $12 \mathrm{~s}$, respectively (Figure 1c). In terms of effective nanostructures dimensions, here, the nominal initial nanopillars radius was $500 \mathrm{~nm}$, and the interpillar distance was $1.5 \mu \mathrm{m}$. However, the newly shaped nanostructures have been characterized by the mean roundness defined as the aspect ratio (AR) between the minor (Am) and the major axis (AM) of the elongated bars. Here, we found that this ratio decreases almost linearly with the exposure 
time (Figure 1d). For our purpose, we chose a $5 \mathrm{~s}$ illumination, which gave the best results in terms of elongation and separation of azobars with an $\mathrm{AR} \approx 0.5$.

Light-induced deformation of azopillars can also be achieved using green light from a confocal microscope. Atomic force microscopy (AFM) was used to characterize the morphology of azostructures before and after confocal light illumination (Figure 2a). AFM measurements show the transformation of a cylindrical pillar (500 $\mathrm{nm}$ in radius) array to an elliptical bar array (3000 $\mathrm{nm}$ length in major axis) after 3 min illumination under a confocal microscope. The resulting profiles show that the ends of the elliptical bars are much narrower than the middle, with the width about $460 \pm 50 \mathrm{~nm}$. By changing the pillar section from circular to elliptical, we also induce a gradual change of the radius of curvature at the ends of azobars. As we show later, these ends are locations where the cell membrane has the highest curvature. AFM measurements of the 3D profiles reveal that these azopillars have vertical walls before light illumination (Figure $2 \mathrm{~b}$ ). The light illumination not only induced nanostructure elongation but also simultaneously induced a decrease in height and softening of the sidewalls (Figure 2c). Next, we demonstrated that it is possible to selectively control and tune the reshaping on individual azopolymer pillars by using a confocal microscope light at $514 \mathrm{~nm}$. By scanning inside a region-of interest centered around a single azopillar, we selectively deformed the azopillar to the bar shape within an array of pillars (Figure 2d). This method can be used to modify locally a tension of a plasma membrane in a particular region of a single cell. Nevertheless, in this work, we focus on the reshaping of a large area of pillars to remodel the cell membrane's architecture of cell population.

\section{Curvature-Dependent Actin Polymerization Can Be Induced by Light-Driven Reshaping of Azopolymer Structures.}

We first confirmed that azopillars and the reshaped azobars are compatible with live cell culture (Figure S1 in the Supporting Information), in agreement with previous reports on azopolymer biocompatibility. ${ }^{29,46}$ To confirm that light driven reshaping of azopolymer structures is able to induce curvature-dependent intracellular responses, we probe the distribution of cellular fibrous actin (F-actin) in response to the presence of different 3D structures. Our recent studies show that static $\mathrm{SiO}_{2}$ nanobar induces high membrane curvatures at its ends while relatively flat membrane along its side walls. F-Actin preferentially accumulates at the ends in correspondence of high membrane curvatures. ${ }^{16,19}$ Here, cells were transfected with LifeAct-RFP for live-cell fluorescence imaging of F-actin. Static $\mathrm{SiO}_{2}$ pillars (500 nm in diameter and $1 \mu \mathrm{m}$ in height) and bars (200 nm in width, 2000 $\mathrm{nm}$ in length, $1 \mu \mathrm{m}$ in height) nanostructures are used as positive controls. Live-cell imaging shows that F-actin forms a ring-structure around $\mathrm{SiO}_{2}$ nanopillars but exhibits strong fluorescence signal at the ends of nanobars with much low signal along the sidewalls of nanobars (Figure 3a). The strong accumulation of F-actin at the end of $\mathrm{SiO}_{2}$ nanobars confirms our recent observations that high membrane curvatures at the ends of nanobars induce local polymerization of F-actin. ${ }^{18}$

Furthermore, we observed that $\mathrm{F}$-actin forms a ring-structure also around azopillars as shown in Figure 3b, while it shows strong accumulation at the two ends of light-reshaped azobars, a phenomenon similar to the accumulation of F-actin at the ends of $\mathrm{SiO}_{2}$. This 
result demonstrates that light-reshaped azobars are indeed able to induce curvaturedependent F-actin formation similar to static $\mathrm{SiO}_{2}$ nanobars. Quantification of the F-actin end-to-side ratios on bar structures shows that the preferential accumulation on the ends of azobars $(1.21 \pm 0.04)$ is less than that on $\mathrm{SiO}_{2}$ nanobars $(2.1 \pm 0.1)$ (Figure $3 \mathrm{c}$ ). This is due to the fact that the size of azobar ends $(\sim 400 \mathrm{~nm})$ is much larger than that of $\mathrm{SiO}_{2}$ nanobars $(200 \mathrm{~nm})$, which results in a lower curvature and less F-actin accumulation. Furthermore, it is worthy to note that the pillar height is reduced depending on the elongation state of the pillars, which can further explain the decrease of fluorescence intensity of F Actin on azobars respect to $\mathrm{SiO}_{2}$ bars.

Furthermore, we probe the spatial distribution of actin nucleation factor Arp2/3 on azobars and $\mathrm{SiO}_{2}$ nanobars. Our previous study showed that branched actin was nucleated by the Arp2/3 complex, ${ }^{16,18,19,47}$ rather than linear actin, accumulated on nanostructures in a curvature-dependent manner. If branched, actin accumulates at high curvature locations; thus, we expect its nucleation factor also accumulates at the same locations. For this study, U2OS cells were transfected to express Arp3-GFP, a subunit of the Arp2/3 complex. Indeed, we observed an accumulation of Arp3-GFP at the ends of both static $\mathrm{SiO}_{2}$ nanobars and light-reshaped azobars as shown in Figure 3d. Overall, these results demonstrate that azobars obtained by light-induced reshaping of azopillars are able to induce curvature-dependent recruitment of Apr2/3 and the subsequent polymerization of F-actin similar to static $\mathrm{SiO}_{2}$ nanobars.

\section{Light-Driven Reshaping of Azostructures Enables Kinetic Measurements of Curvature- Dependent F-Actin Polymerization in Live Cells.}

The dynamic nature of the light-induced reshaping of azopolymer structures allows kinetic measurements of curvature-dependent F-actin polymerization. For this measurement, we cultured cells for $24 \mathrm{~h}$ and observed the accumulation of F-actin on azopillars (Figure 4a). Then the $532 \mathrm{~nm}$ laser $(1.5 \mathrm{~mW})$ was used to induce the azopolymer nanostructure array, reshaping in the presence of live cells. Here, we set a $5 \mathrm{~s}$ exposure time as we observed $\sim 100 \%$ cell viability. Longer exposure time results in the ends of neighboring azobars too close to each other, in which case the cell membrane could not fully wrap around individual azobars. Also, phototoxicity clearly increased with exposure time 15 s or longer (Figure S1 in the Supporting Information).

By taking a time series of Lifeact-GFP snapshots of the selected cell, we measure the dynamics of F-actin reorganization after green-light induced azopolymer reshaping. For fluorescence imaging, we used a weak blue light $(0.1 \mathrm{~mW})$ that does not cause azopolymer reshaping. Figure $4 \mathrm{~b}$ shows fluorescence images of LifeAct-GFP around a single azobar at different times after the reshaping. These images clearly demonstrate F-actin transitioning from a ring-like conformation after $5 \mathrm{~min}$ to a definitely distributed and preferential accumulation to the bar ends after $15 \mathrm{~min}$. Figure 4c shows the fluorescence intensity plot where Lifeact-GFP along the sidewall and a concurrent increase toward the ends of reshaped pillars around $10 \mathrm{~min}$ from topographic reshaping. On average, the F-actin reorganization happens around $10 \pm 2$ min after the laser-induced deformation. Therefore, dynamic 
reshaping of azopolymer topography allows us to monitor kinetics of curvature-sensitive actin polymerization in real-time.

In response to abrupt external perturbations, cells exhibit both elastic and viscous behavior influenced by a combination of membrane, actin cortex, and cytoplasm. The membrane response is highly nonlinear, and the time scale ranges from milliseconds to tens of seconds depending on the time scale and the amplitude of the perturbation. ${ }^{48}$ The subsequent cytoskeleton reorganization takes longer time. The time scale we observed for actin cytoskeleton remodeling is consistent with previous studies of cellular processes that rely on cytoskeletal remodeling. ${ }^{49-52}$

\section{Conclusion.}

In this work, we demonstrate the possibility to use light-responsive azopolymer nanostructures to dynamically change membrane curvatures and intracellular curvature dependent processes in live cells. We note that dynamically modulating membrane curvatures has been previously achieved by pushing or pulling the cell membrane using optical tweezers and atomic force microscopy. ${ }^{42,43}$ Compared with optical tweezers or AFM, the azopolymer deformation approach is fundamentally different and has unique advantages. It affords high throughput, easy access, and does not require sophisticated skills or instruments. Using polarized laser beam, we are able to switch pillar to bar shape along the polarization direction, which dynamically modulates the membrane curvature of U2OS cells to activate intracellular curvature-sensing processes. We demonstrate that curvature dependent F-actin reorganization takes about $10 \mathrm{~min}$ after the light-induced azopolyer reshaping. In this work, the photo responsive property of azopolymer provides unique advantages for dynamically modulating cellular activities.

\section{Supplementary Material}

Refer to Web version on PubMed Central for supplementary material.

\section{ACKNOWLEDGMENTS}

We thank Jong Wook Noh for the support of the fabrication process, Dr. Carolyn Bertozzi for the confocal microscope, and Stanford Nanofabrication and Characterization facilities for the use of instrument. This work was supported by NIH R01GM128142.

\section{ABBREVIATIONS}

pDR1m poly(dispersed Red 1 methacrylate)

PDMS poly (dimethylsiloxane)

\section{REFERENCES}

(1). Ventre M; Natale CF; Rianna C; Netti PA Topographic Cell Instructive Patterns to Control Cell Adhesion, Polarization and Migration. J. R. Soc., Interface 2014, 11 (100), 20140687. [PubMed: 25253035]

(2). Bettinger CJ; Langer R; Borenstein JT Engineering Substrate Topography at the Micro and Nanoscale to Control Cell Function. Angew. Chem., Int. Ed 2009, 48, 5406-5415. 
(3). Wu Y; Yang Z; Law JBK; He AY; Abbas AA; Denslin V; Kamarul T; Hui JHP; Lee EH The Combined Effect of Substrate Stiffness and Surface Topography on Chondrogenic Differentiation of Mesenchymal Stem Cells. Tissue Eng., Part A 2017, 23 (1-2), 43-54. [PubMed: 27824280]

(4). Brammer KS; Choi C; Frandsen CJ; Oh S; Jin S Hydrophobic Nanopillars Initiate Mesenchymal Stem Cell Aggregation and Osteo-Differentiation. Acta Biomater. 2011, 7 (2), 683-690. [PubMed: 20863916]

(5). Xie C; Hanson L; Xie W; Lin Z; Cui B; Cui Y Noninvasive Neuron Pinning with Nanopillar Arrays. Nano Lett. 2010, 10 (10), 4020-4024. [PubMed: 20815404]

(6). Persson H; Købler C; Mølhave K; Samuelson L; Tegenfeldt JO; Oredsson S; Prinz CN Nanowires: Fibroblasts Cultured on Nanowires Exhibit Low Motility, Impaired Cell Division, and DNA Damage (Small 23/2013). Small 2013, 9 (23), 3905.

(7). Sun J; Ding Y; Lin NJ; Zhou J; Ro H; Soles CL; Cicerone MT; Lin-Gibson S Exploring Cellular Contact Guidance Using Gradient Nanogratings. Biomacromolecules 2010, 11 (11), 3067-3072. [PubMed: 20954734]

(8). Wu Y-N; Law JBK; He AY; Low HY; Hui JHP; Lim CT; Yang Z; Lee EH Substrate Topography Determines the Fate of Chondrogenesis from Human Mesenchymal Stem Cells Resulting in Specific Cartilage Phenotype Formation. Nanomedicine 2014, 10 (7), 1507-1516. [PubMed: 24768908]

(9). Sniadecki NJ; Desai RA; Ruiz SA; Chen CS Nanotechnology for Cell-Substrate Interactions. Ann. Biomed. Eng 2006, 34 (1), 59-74. [PubMed: 16525764]

(10). Antonini S; Meucci S; Parchi P; Pacini S; Montali M; Poggetti A; Lisanti M; Cecchini M Human Mesenchymal Stromal Cell-Enhanced Osteogenic Differentiation by Contact Interaction with Polyethylene Terephthalate Nanogratings. Biomed. Mater 2016, 11 (4), 045003. [PubMed: 27388559]

(11). Jung AR; Kim RY; Kim HW; Shrestha KR; Jeon SH; Cha KJ; Park YH; Kim DS; Lee JY Nanoengineered Polystyrene Surfaces with Nanopore Array Pattern Alters Cytoskeleton Organization and Enhances Induction of Neural Differentiation of Human Adipose-Derived Stem Cells. Tissue Eng., Part A 2015, 21 (13-14), 2115-2124. [PubMed: 25919423]

(12). Lavenus S; Berreur M; Trichet V; Pilet P; Louarn G; Layrolle P Adhesion and Osteogenic Differentiation of Human Mesenchymal Stem Cells on Titanium Nanopores. Eur. Cell. Mater 2011, 22, 84-96. [PubMed: 21870339]

(13). Dalby MJ; Gadegaard N; Tare R; Andar A; Riehle MO; Herzyk P; Wilkinson CDW; Oreffo ROC The Control of Human Mesenchymal Cell Differentiation Using Nanoscale Symmetry and Disorder. Nat. Mater 2007, 6 (12), 997-1003. [PubMed: 17891143]

(14). Chen S; Jones JA; Xu Y; Low H-Y; Anderson JM; Leong KW Characterization of Topographical Effects on Macro phage Behavior in a Foreign Body Response Model. Biomaterials 2010, 31 (13), 3479-3491. [PubMed: 20138663]

(15). Luu TU; Gott SC; Woo BWK; Rao MP; Liu WF Micro and Nanopatterned Topographical Cues for Regulating Macrophage Cell Shape and Phenotype. ACS Appl. Mater. Interfaces 2015, 7 (51), 28665-28672. [PubMed: 26605491]

(16). Zhao W; Hanson L; Lou H-Y; Akamatsu M; Chowdary P; Santoro F; Marks J; Grassart A; Drubin D; Cui Y Nanoscale Manipulation Of Membrane Curvature For Probing Endocytosis In Live Cells. Nat. Nanotechnol 2017, 12, 750. [PubMed: 28581510]

(17). Galic M; Jeong S; Tsai F-C; Joubert L-M; Wu YI; Hahn KM; Cui Y; Meyer T External Push and Internal Pull Forces Recruit Curvature-Sensing N-BAR Domain Proteins to the Plasma Membrane. Nat. Cell Biol 2012, 14 (8), 874-881. [PubMed: 22750946]

(18). Lou H-Y; Zhao W; Cui B Membrane Curvature Dependent F-Actin Polymerization at Nano-Cell Interface. Biophys. J 2018, 114, 690a.

(19). Lou H-Y; Zhao W; Zeng Y; Cui B The Role of Membrane Curvature in Nanoscale TopographyInduced Intracellular Signaling. Acc. Chem. Res 2018, 51 (5), 1046-1053. [PubMed: 29648779]

(20). Azatov M; Sun X; Suberi A; Fourkas JT; Upadhyaya A Topography on a Subcellular Scale Modulates Cellular Adhesions and Actin Stress Fiber Dynamics in Tumor Associated Fibroblasts. Phys. Biol 2017, 14, 065003. [PubMed: 28635615] 
(21). Li X; Matino L; Zhang W; Klausen L; McGuire AF; Lubrano C; Zhao W; Santoro F; Cui B A Nanostructure Platform for Live-Cell Manipulation of Membrane Curvature. Nat. Protoc 2019, 14 (6), 1772-1802. [PubMed: 31101905]

(22). Beckwith KS; Cooil SP; Wells JW; Sikorski P Tunable High Aspect Ratio Polymer Nanostructures for Cell Interfaces. Nanoscale 2015, 7 (18), 8438-8450. [PubMed: 25891641]

(23). Lou H-Y; Zhao W; Li X; Duan L; Powers A; Akamatsu M; Santoro F; McGuire AF; Cui Y; Drubin DG; et al. Membrane Curvature Underlies Actin Reorganization in Response to Nanoscale Surface Topography. Proc. Natl. Acad. Sci. U. S. A 2019, 116 (46), 23143-23151. [PubMed: 31591250]

(24). Dipalo M; McGuire AF; Lou H-Y; Caprettini V; Melle G; Bruno G; Lubrano C; Matino L; Li X; De Angelis F; et al. Cells Adhering to 3D Vertical Nanostructures: Cell Membrane Reshaping without Stable Internalization. Nano Lett. 2018, 18 (9), 6100-6105. [PubMed: 30091365]

(25). Gopal S; Chiappini C; Penders J; Leonardo V; Seong H; Rothery S; Korchev Y; Shevchuk A; Stevens MM Porous Silicon Nanoneedles Modulate Endocytosis to Deliver Biological Payloads. Adv. Mater 2019, 31 (12), No. e1806788. [PubMed: 30680803]

(26). Beckwith KS; Ullmann S; Vinje J; Sikorski P Influence of Nanopillar Arrays on Fibroblast Motility, Adhesion, and Migration Mechanisms. Small 2019, 15 (43), No. e1902514. [PubMed: 31464377]

(27). Zhao W; Hanson L; Lou H-Y; Akamatsu M; Chowdary PD; Santoro F; Marks JR; Grassart A; Drubin DG; Cui Y; et al. Nanoscale Manipulation of Membrane Curvature for Probing Endocytosis in Live Cells. Nat. Nanotechnol 2017, 12 (8), 750-756. [PubMed: 28581510]

(28). Higgins SG; Becce M; Seong H; Stevens MM Nanoneedles and Nanostructured Surfaces for Studying Cell Interfacing In International Conference on the Development of Biomedical Engineering in Vietnam; Springer, 2018; pp 209-212.

(29). Pirani F; Angelini A; Frascella F; Rizzo R; Ricciardi S; Descrovi E Light-Driven Reversible Shaping of Individual Azopolymeric Micro-Pillars. Sci. Rep 2016, 6, 31702. [PubMed: 27531219]

(30). Wang W; Yao Y; Luo T; Chen L; Lin J; Li L; Lin S Deterministic Reshaping of Breath Figure Arrays by Directional Photomanipulation. ACS Appl. Mater. Interfaces 2017, 9 (4), 4223-4230. [PubMed: 28071893]

(31). Pirani F; Angelini A; Frascella F; Descrovi E Reversible Shaping of Microwells by Polarized Light Irradiation. Int. J. Polym. Sci 2017, 2017, 1.

(32). Kong X; Wang X; Luo T; Yao Y; Li L; Lin S Photomanipulated Architecture and Patterning of Azopolymer Array. ACS Appl. Mater. Interfaces 2017, 9 (22), 19345-19353. [PubMed: 28504513]

(33). Cojocariu C; Rochon P Light-Induced Motions in Azobenzene-Containing Polymers. Pure Appl. Chem 2004, 76, 1479-1497.

(34). Bandara HMD; Dhammika Bandara HM; Burdette SC Photoisomerization in Different Classes of Azobenzene. Chem. Soc. Rev 2012, 41, 1809-1825. [PubMed: 22008710]

(35). Yadavalli NS; Loebner S; Papke T; Sava E; Hurduc N; Santer S A Comparative Study of Photoinduced Deformation in Azobenzene Containing Polymer Films. Soft Matter 2016, 12, 2593-2603. [PubMed: 26853516]

(36). Lee S; Kang HS; Ambrosio A; Park J-K; Marrucci L Directional Superficial Photofluidization for Deterministic Shaping of Complex 3D Architectures. ACS Appl. Mater. Interfaces 2015, 7, 8209-8217. [PubMed: 25816857]

(37). Lee S; Shin J; Lee Y-H; Fan S; Park J-K Directional Photofluidization Lithography for Nanoarchitectures with Controlled Shapes and Sizes. Nano Lett. 2010, 10, 296-304. [PubMed: 20017565]

(38). Kang HS; Lee S; Park J-K Monolithic, Hierarchical Surface Reliefs by Holographic Photofluidization of Azopolymer Arrays: Direct Visualization of Polymeric Flows. Adv. Funct. Mater 2011, 21, 4412-4422.

(39). Pirani F; Angelini A; Ricciardi S; Frascella F; Rizzo R; Ferrarese Lupi F; De Leo N; Boarino L; Descrovi E Tunable Hydrophobicity Assisted by Light-Responsive Surface Micro Structures. Laser-based Micro- and Nanoprocessing XI 2017, 100920A DOI: 10.1117/12.2251008. 
(40). Oscurato SL; Borbone F; Maddalena P; Ambrosio A Light-Driven Wettability Tailoring of Azopolymer Surfaces with Reconfigured Three-Dimensional Posts. ACS Appl. Mater. Interfaces 2017, 9, 30133-30142. [PubMed: 28805057]

(41). Pirani F; Angelini A; Ricciardi S; Frascella F; Descrovi E Laser-Induced Anisotropic Wettability on Azopolymeric Micro Structures. Appl. Phys. Lett 2017, 110, 101603.

(42). Nussenzveig HM Cell Membrane Biophysics with Optical Tweezers. Eur. Biophys. J 2018, 47 (5), 499-514. [PubMed: 29164289]

(43). Haase K; Pelling AE Investigating Cell Mechanics with Atomic Force Microscopy. J. R. Soc., Interface 2015, 12 (104), 20140970. [PubMed: 25589563]

(44). Schaumann EN; Tian B Actin-Packed Topography: Cytoskeletal Response to Curvature. Proc. Natl. Acad. Sci. U. S. A 2019, 116 (46), 22897-22898. [PubMed: 31645378]

(45). Rianna C; Rossano L; Kollarigowda RH; Formiggini F; Cavalli S; Ventre M; Netti PA Dynamic Cell Substrates: Spatio Temporal Control of Dynamic Topographic Patterns on Azopolymers for Cell Culture Applications (Adv. Funct. Mater. 42/2016). Adv. Funct. Mater 2016, 26, 7743.

(46). Rianna C; Rossano L; Kollarigowda RH; Formiggini F; Cavalli S; Ventre M; Netti PA SpatioTemporal Control of Dynamic Topographic Patterns on Azopolymers for Cell Culture Applications. Adv. Funct. Mater 2016, 26 (42), 7572-7580.

(47). Hanson L; Zhao W; Lou H-Y; Lin ZC; Lee SW; Chowdary P; Cui Y; Cui B Vertical Nanopillars for in Situ Probing of Nuclear Mechanics in Adherent Cells. Nat. Nanotechnol 2015, 10, 554562. [PubMed: 25984833]

(48). Kollmannsberger P; Fabry B Linear and Nonlinear Rheology of Living Cells. Annu. Rev. Mater. Res 2011, 41 (1), 75-97.

(49). Fung TS; Ji W-K; Higgs HN; Chakrabarti R Two Distinct Actin Filament Populations Have Effects on Mitochondria, with Differences in Stimuli and Assembly Factors. J. Cell Sci 2019, 132 (18), jcs234435. [PubMed: 31413070]

(50). Chierico L; Joseph AS; Lewis AL; Battaglia G Live Cell Imaging of Membrane/Cytoskeleton Interactions and Membrane Topology. Sci. Rep 2015, 4, 6056.

(51). Fritzsche M; Fernandes RA; Chang VT; Colin-York H; Clausen MP; Felce JH; Galiani S; Erlenkamper C; Santos AM; Heddleston JM; et al. Cytoskeletal Actin Dynamics Shape a Ramifying Actin Network Underpinning Immunological Synapse Formation. Sci. Adv 2017, 3 (6), No. e1603032. [PubMed: 28691087]

(52). Lee S-L; Nekouzadeh A; Butler B; Pryse KM; McConnaughey WB; Nathan AC; Legant WR; Schaefer PM; Pless RB; Elson EL; et al. Physically-Induced Cytoskeleton Remodeling of Cells in Three-Dimensional Culture. PLoS One 2012, 7 (12), No. e45512. [PubMed: 23300512] 
(a)

(c)

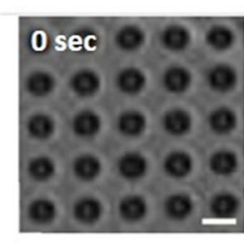

\section{Figure 1.}

(b)
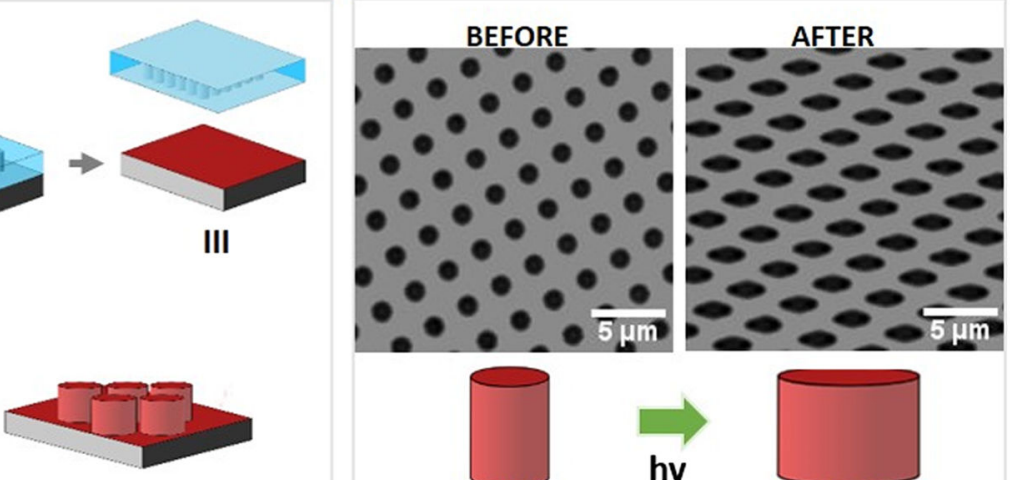

V

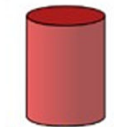

CIRCULAR NANOPILLAR

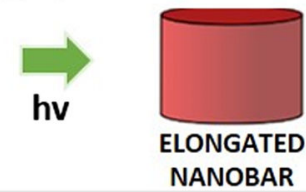

Light-induced reshaping of azopolymer 3D structures. (a) Schematics of azopolymer pillar fabrication and light induced shape deformation; (I) silicon master fabricated by e-beam lithography, (II) replica of the PDMS mold, (III) soft imprinting of the PDMS mold on a thin film of pDR1m azopolymer for making azopolymer pillars. Imprinted azopillar array (IV) can be reshaped into azobar array (V) after light illumination. (b) Brightfield images of azopolymer pillars before and after a green laser-induced deformation. Scale bar $5 \mu \mathrm{m}$. Lower part, schematic of single pillar-to-bar deformation by light illumination. (c) Brightfield images of polymer pillars at different exposure time. Pillar transit from pillar $(0 \mathrm{~s})$, to slightly elongated (4 s), to bar-like structures (8-12 s). Scale bar $2 \mu \mathrm{m}$. (d) Aspect ratio value $(\mathrm{AR}=\mathrm{Am} / \mathrm{AM})$ for pillars during laser irradiation from 0 to $12 \mathrm{~s}$. Minor axes $(\mathrm{Am})$ are represented in black and major (AM) in red. 
(a)

(c)
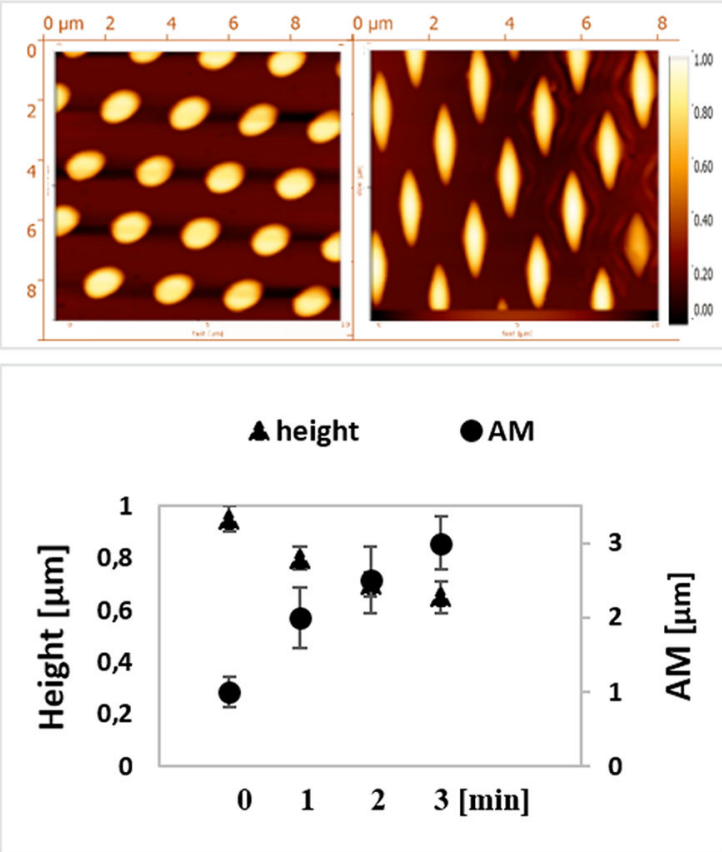

Figure 2. Scale bar $10 \mu \mathrm{m}$. (b)

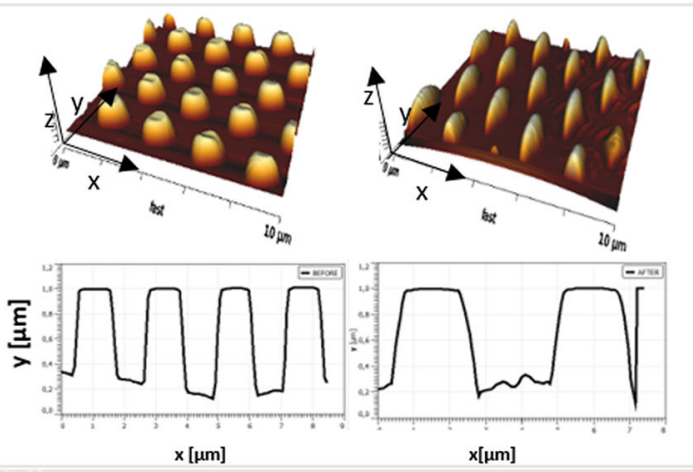

(d)

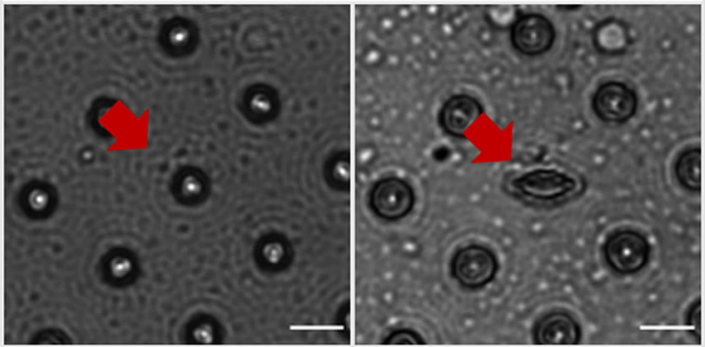

AFM characterization of light-reshaped individual azostructures. (a) AFM topographic images show the transformation of circular pillar (500 $\mathrm{nm}$ radius) arrays to elliptical bar arrays (3000 $\mathrm{nm}$ length in major axis) under a confocal microscope. (b) Three-dimensional AFM measurements and section profiles of azopillars and light-reshaped azobars. (c) Plot of height (triangle) and major axes length (circle) of elongated pillars over time (0-3 min). (d) Brightfield images of a single azopillar selectively reshaped by a $514 \mathrm{~nm}$ laser under a confocal microscope. A region of interest (ROI) is defined around the selected azopillar. 

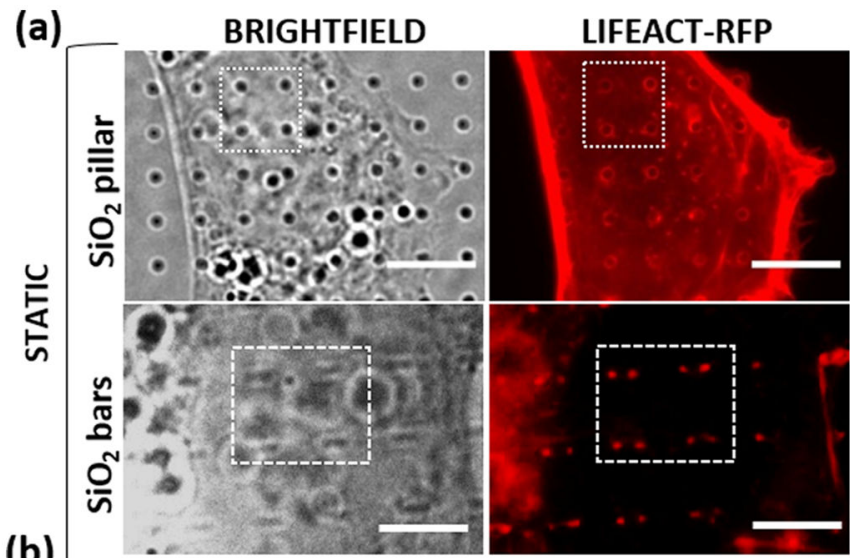

\section{ZOOM-IN}

(b)
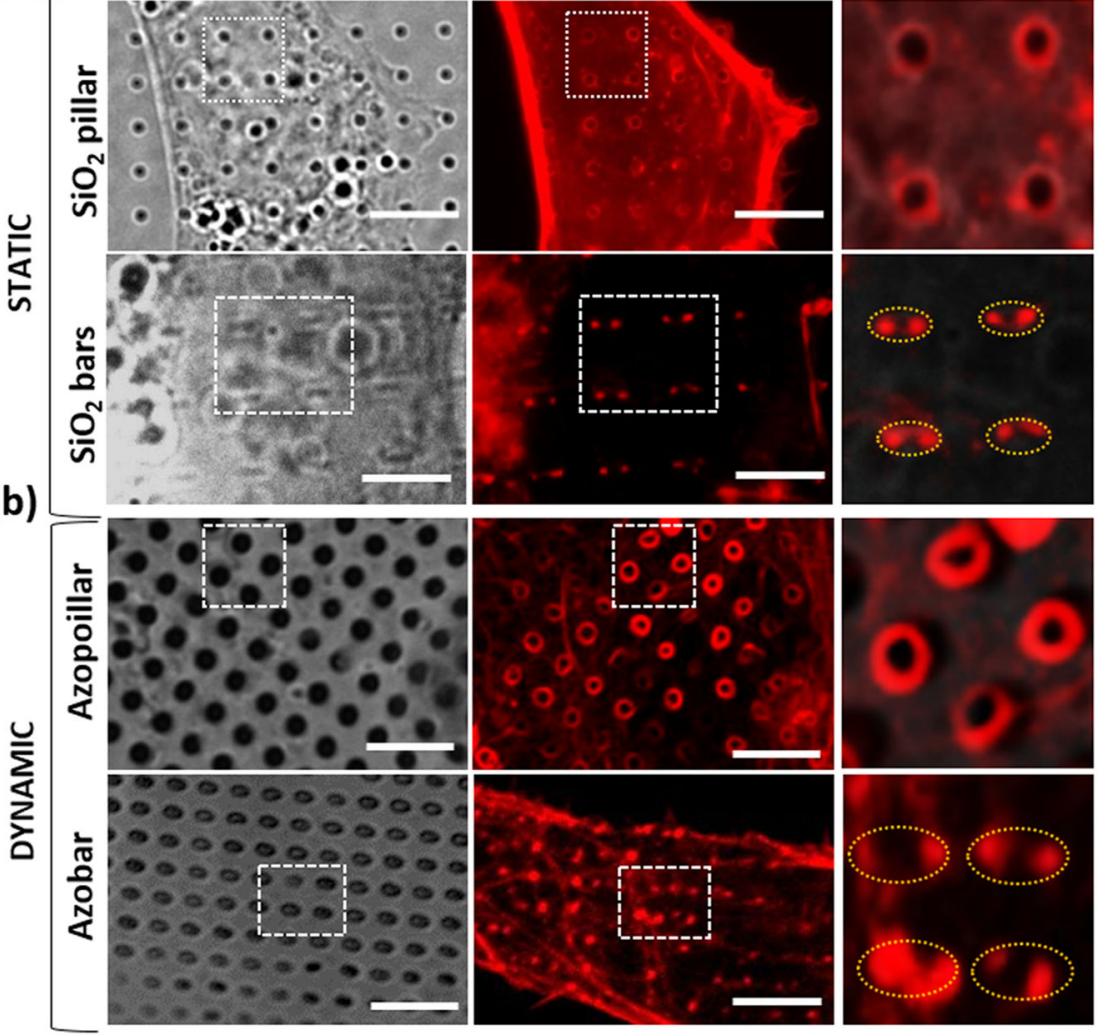

(c)
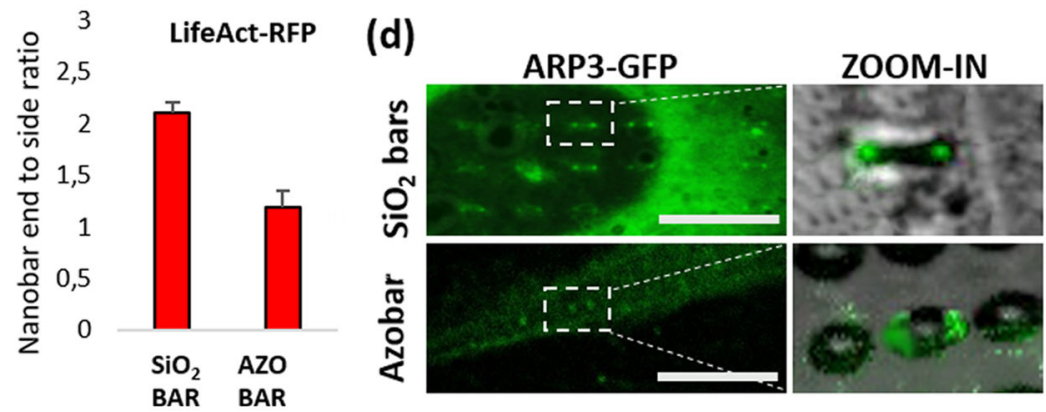

Figure 3.

Azobars induce curvature-dependent actin polymerization. (a) Brightfield and fluorescence images of U2OS cells transfected with Lifeact-RFP on static $\mathrm{SiO}_{2}$ structures. Lifeact-RFP appears as a ring around nanopillars and preferentially accumulates to the ends of $\mathrm{SiO}_{2}$ bars. Scale bar $10 \mu \mathrm{m}$. (b) Brightfield and fluorescence images of U2OS cells transfected with Lifeact-RFP on azostructures. Similar to $\mathrm{SiO}_{2}$ nanostructures, Lifeact RFP appears as a ring around azopillars and appears as two spots at the ends of azobars. Scale bar $10 \mu \mathrm{m}$. (c) Quantification of nanobar end-to-side ratios for Lifeact-RFP on $\mathrm{SiO}_{2}$ bars and azobars ( $n=$ 10 cells for each condition). Error bars represent s.e.m. (d) Fluorescence images of U2OS cells expressing Arp3-GFP show strong preference of Arp3-GFP to the ends of $\mathrm{SiO}_{2}$ nanobars as well as azobars. Scale bar $10 \mu \mathrm{m}$. 


\section{(a)}
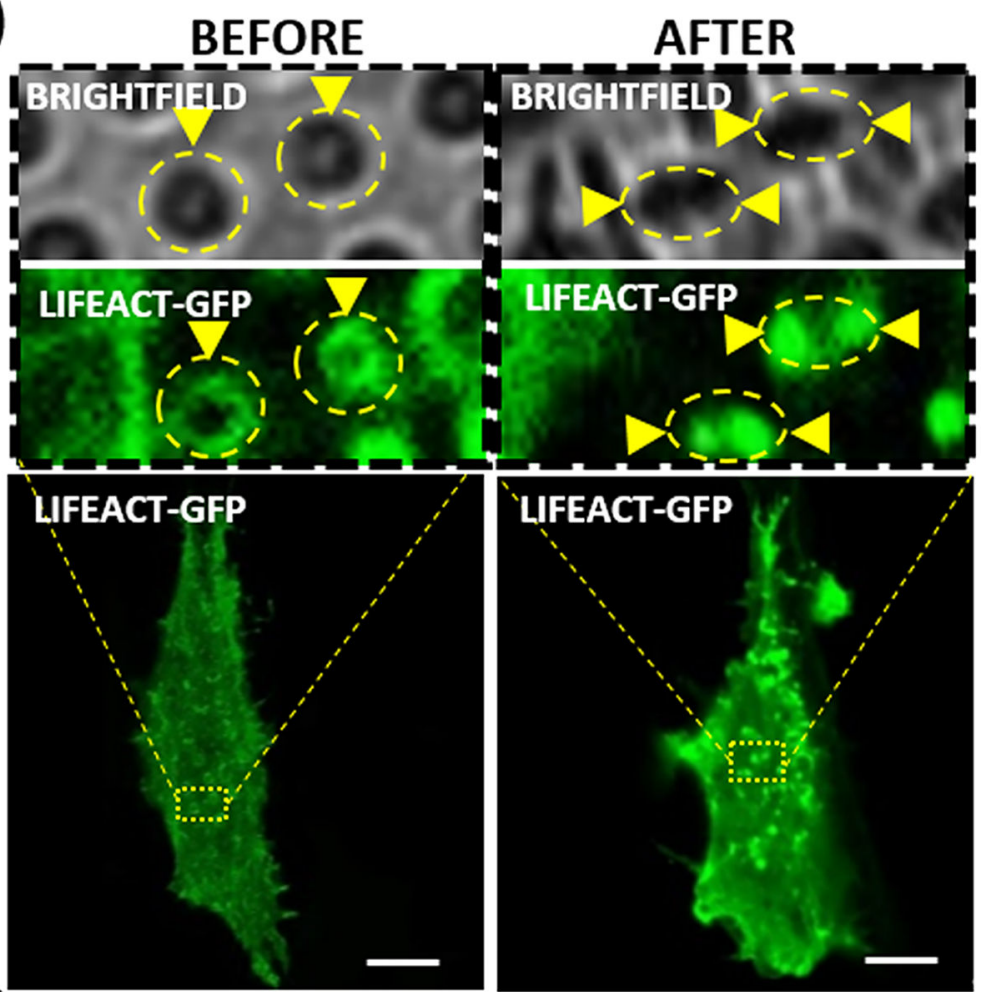

(b)
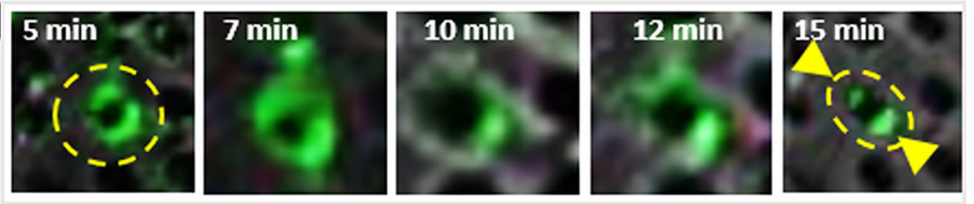

(c)
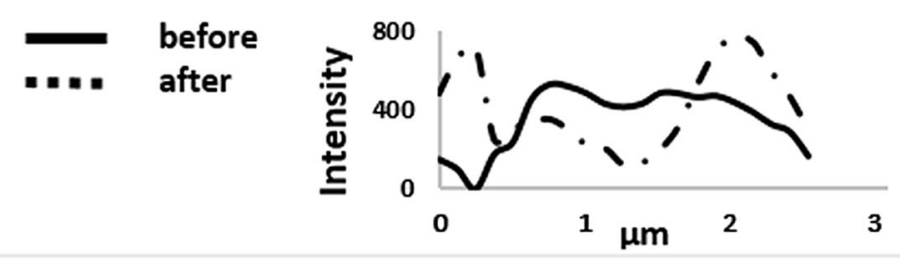

Figure 4.

Monitoring curvature-dependent F-actin polymerization in live cells by light-driven reshaping of azostructures. (a) Fluorescence images of U2OS cells expressing Lifeact-GFP before and after $\sim 10 \mathrm{~min}$ from azopillar reshaping. The accumulation of F-actin is observed around pillar and at the ends of bars with the high magnification image (dashed circle). (b) High-magnification images of the same azobar at different times show the reorganization of F-actin in response to azostructure reshaping. (c) Intensity profiles of LifeAct-GFP along reshaped pillar, before illumination at 0 min (black line) and 10 min after stimulation (dashed line). The intensity distribution is measured in the same selected ROI and calculated over time with ImageJ. 